\title{
TOWARDS DEVELOPING A MICROPULSE DIFFERENTIAL ABSORPTION LIDAR TO MEASURE ATMOSPHERIC TEMPERATURE
}

\author{
Robert A. Stillwell ${ }^{1,2, *}$, Scott M. Spuler ${ }^{2}$, Matthew Hayman², Catharine E. Bunn ${ }^{3}$, and Kevin S. \\ Repasky ${ }^{2,3,4}$ \\ ${ }^{1}$ Advanced Study Program, National Center for Atmospheric Research, Boulder, CO 80301, USA. \\ ${ }^{2}$ Earth Observing Laboratory, National Center for Atmospheric Research, Boulder, CO 80301, USA. \\ ${ }^{3}$ Physics Department, Montana State University, Bozeman, MT 59717, USA. \\ ${ }^{4}$ Electrical and Computer Engineering, Montana State University, Bozeman, MT 59717, USA. \\ *Email: stillwel@ucar.edu
}

\begin{abstract}
It has generally been assumed that differential absorption lidar (DIAL) systems are incapable of measuring atmospheric temperature with useful accuracy. This assumption is a direct result of errors that arise in standard DIAL retrievals due to differential Rayleigh-Doppler broadening from aerosols and molecules. We present here, a combined high spectral resolution (HSRL) and DIAL system that addresses this identified source of uncertainty by measuring quantitative aerosol parameters as well as oxygen absorption parameters. This system, in combination with a perturbative retrieval method, accounts for the Rayleigh-Doppler broadening effects on the oxygen absorption. We describe this combined DIAL/HSRL system and retrieval to evaluate the first retrieval parameters exploring the likelihood that it is possible to measure atmospheric temperature using a DIAL system.
\end{abstract}

\section{INTRODUCTION}

Measurements of thermodynamic variables, such as temperature and water vapor mixing-ratio, are critical to understanding and predicting the state of the atmosphere. Much of the predictive skill of numerical weather forecasting is related to the knowledge of these variables in both space and time. Twice daily, hundreds of radiosondes are released all over the globe, which are assimilated into operational weather forecasting systems, to specify the thermodynamic state of the atmosphere and guide weather prediction. This system has proven effective but is limited in application. The infrastructure needed to launch hundreds of sondes simultaneously is fundamentally limited, especially regarding temporal resolution. Many reports have called for an improvement in this atmospheric measurement system [1-5]. Lidar systems show promise to dramatically increase the temporal resolution of thermodynamic profiles, but current temperature lidar systems are not well suited for wide unattended network deployment.

An ongoing collaboration between Montana State University (MSU) and the National Center for Atmospheric Research (NCAR) has resulted in the advancement of diode-laser-based (DLB) lidar systems that are used to perform eye-safe, continuous, and unattended measurements of the atmosphere over the time period of several weeks to months [6-9]. This DLB architecture is adaptable to a number of relevant lidar system types. A prototype water vapor differential absorption lidar (DIAL) has been described and validated $[9,10]$. Additionally, a high spectral resolution lidar (HSRL) system based on a rubidium vapor cell has been demonstrated [11]. Together, a combination of DIAL and HSRL along with a perturbative retrieval shows promise to measure atmospheric temperature [12].

Previous attempts to measure atmospheric temperature using DIAL leveraging absorption in the oxygen A-band have highlighted major error sources due to differential Rayleigh-Doppler broadening between aerosol and molecular scatterers [13]. The absorption of light after scattering is fundamentally a function of the ratio of total scattering to molecular scattering, i.e. the backscatter ratio. It was proposed, therefore, that a Klett type inversion might suffice using the DIAL offline wavelength to retrieve the backscatter ratio, but this failed to yield useful accuracy - errors on the order of $10^{\circ} \mathrm{C}$ were observed [13].

We have demonstrated a DIAL system that uses the oxygen A-band and retrieves aerosol information from an integrated HSRL. This data 
is then used with a perturbative retrieval to retrieve temperature. This system uses a potassium vapor cell with the $\mathrm{D}_{1}$ absorption line acting as a narrow band (aerosol blocking) filter. This presentation will describe the system and the initial measurement results and progress towards making an autonomous DLB system capable of measuring full atmospheric thermodynamic profiles.

\section{LIDAR HARDWARE DESIGN}

The measurement of temperature using DIAL relies on the availability of three high quality measurements: oxygen differential absorption, water vapor number density, and the backscatter ratio. Using absorption measurements of a wellmixed species like oxygen forms the basis of the measurement technique. If not accounted for, the exact mixing-ratio of oxygen can be sufficiently altered by the water vapor mixing-ratio to bias the retrieval. Additionally, the backscatter ratio must be known at all altitudes in common volume with the DIAL measurements to correct for the differential Rayleigh-Doppler broadening of the backscattered light in the temperature retrieval. Figure 1 shows a block diagram of a combined DIAL/HSRL system capable of making all three measurements.

Figure 1 shows a combined two-species DIAL system. The first, highlighted in gray, is the WV DIAL system described previously [9]. The red boxes show the needed additions to measure temperature. The transmitters are, by design, identical except their output wavelength. The receivers are similar as well, with an addition of a potassium gas vapor cell in the oxygen receiver channel.

Several design features are of note that allow for the measurement of atmospheric temperature in an unattended, field-deployable, and eye-safe manner. At its core, the system described in Figure 1 is an oxygen DIAL system leveraging the oxygen Aband. A pair of Distributed Bragg Reflectors (DBRs) are used, which are fiber coupled. A set of opto-electric switches interleaves the outgoing pulses at a rate of $100 \mathrm{~Hz}$. Both lasers use a common amplifier, which, in combination with the receiver design, allows for the removal of all overlap corrections. The water vapor and oxygen DIAL beams are combined with a dichroic beam splitter and use a shared telescope design to transmit the beam to the environment. This design provides needed passive opto-mechanical stability for long term unattended operation.

The receiver for the combined system is fiber coupled by a first-stage receiver, providing longterm stability and simplifying alignment. The second-stage receiver for both water vapor and oxygen consist of interference filters, with full widths at half maximum (FWHM) of approximately $0.5 \mathrm{~nm}$ and $1 \mathrm{~nm}$, respectively. The filters provide high out of band blocking, on the order of OD 12. For the WV system, the filters are 2 identical custom filters made by Alluxa. For the oxygen system, a single $13 \mathrm{~nm}$ FWHM filter from Semrock (part number FF01-766/13-25) and a single $1 \mathrm{~nm}$ FWHM filter from Aluxa (part number C11-3268-7270-769BP \# 102) are used. Both channels also have a custom narrowband solid etalon with FWHM of $1 \mathrm{GHz}$ and $10 \mathrm{GHz}$, respectively. Both designs also pass both on- and offline wavelengths in adjacent longitudinal modes of the etalon to use a common detector for a DIAL wavelength pair.

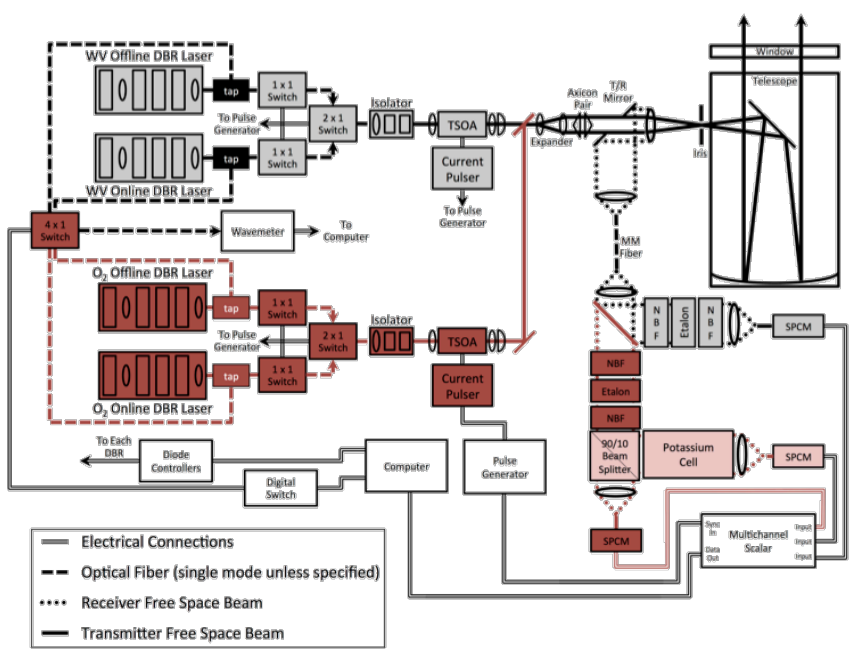

Figure 1: Block diagram of a combined DIAL/HSRL system capable of measuring atmospheric thermodynamic profiles.

Specifications of the combined DIAL/HSRL system are given in Table 1. The wavelengths for water vapor measurements can be changed to increase or decrease the absorption as governed by the prevailing environmental conditions. The 
wavelengths for oxygen DIAL are not as flexible as in the water vapor system, as the offline must be tuned to the potassium $\mathrm{D}_{1}$ line and the online wavelength tuned to the $769.8 \mathrm{~nm}$ oxygen line.

Table 1: Transmitter and Receiver specifications.

\begin{tabular}{|c|c|c|c|}
\hline Transmitter & Spec & Receiver & Spec \\
\hline $\begin{array}{l}\text { Locking } \\
\text { stability [MHz] }\end{array}$ & 25.3 & $\begin{array}{l}\text { Telescope } \\
\text { Diameter }[\mathrm{cm}]\end{array}$ & 40 \\
\hline $\begin{array}{l}\text { Online } \lambda[\mathrm{nm}] \\
\left(\mathrm{WV}, \mathrm{O}_{2}\right)\end{array}$ & $\begin{array}{c}\approx 828.195, \\
769.7958\end{array}$ & $\begin{array}{l}\text { Bandpass [nm] } \\
\left(\mathrm{WV}, \mathrm{O}_{2}\right)\end{array}$ & $\begin{array}{l}0.012, \\
0.080\end{array}$ \\
\hline $\begin{array}{l}\text { Offline } \lambda[\mathrm{nm}] \\
\left(\mathrm{WV}, \mathrm{O}_{2}\right)\end{array}$ & $\begin{array}{c}\approx 828.295 \\
770.1085\end{array}$ & $\begin{array}{l}\text { Field of View } \\
{[\mu \mathrm{rad}]}\end{array}$ & 100 \\
\hline $\begin{array}{l}\text { Power }[\mathrm{mW}] \\
\left(\mathrm{WV}, \mathrm{O}_{2}\right)\end{array}$ & $\begin{array}{l}\approx 25 \\
\approx 17.5\end{array}$ & $\begin{array}{l}\text { Range } \\
\text { Resolution [m] }\end{array}$ & 150 \\
\hline
\end{tabular}

As a result, the oxygen channel etalon's free spectral range is tightly constrained as its transmission lines must align with both the oxygen and potassium lines. A scan of the oxygen receiver etalon with the modeled oxygen spectrum and potassium $\mathrm{D}_{1}$ line are shown in Figure 2.

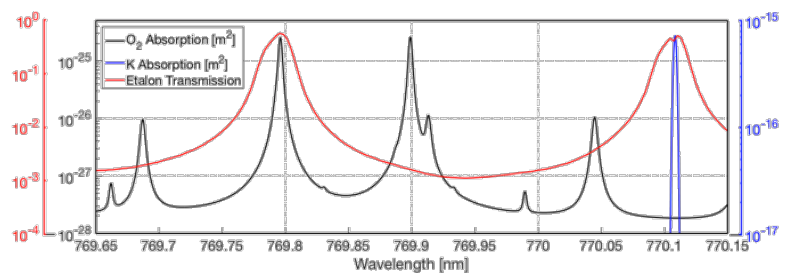

Figure 2: Spectral characteristics of the oxygen Aband, potassium $D_{1}$ line, and etalon. The etalon that passes both DIAL wavelengths was designed with a FSR of $157.90 \mathrm{GHz}$, with measured FSR of 158.13 GHz.

Finally, this etalon design provides a method of easily calculating the HSRL calibration parameters. As the on- and offline wavelengths have the same overlap function and use the same detectors, the online wavelength can be used to calculate the range dependent calibration information. The ratio of the oxygen online raw photons measured on the combined and molecular detectors (so named from the offline HSRL signals observed) yields the calibration constants directly without having to remove optical elements or alter the receiver in any way.

\section{FIRST MEASUREMENTS}

Temperature measurements are made using the above-described system using a retrieval based on a perturbative solution to the two-wavelength DIAL equation [12]. This retrieval takes an initial estimate of the temperature profile to retrieve water vapor and backscatter ratio. We use groundbased weather station temperature and an assumed constant lapse rate. These values are, by design, relatively insensitive to temperature. Using these retrieved water vapor mixing-ratio and backscatter ratio, the temperature-sensitive oxygen absorption coefficient is retrieved. Temperature is retrieved from the oxygen absorption coefficient by using an iterative version of the perturbative retrieval.

First temperature measurements are shown in Figure 3. For context, surface based weather station temperature values are also provided. These data are taken over a clear 38-hour period without the etalon described in Section 2. The surface temperature and first range bin show qualitative agreement, especially highlighting the day-to-night temperature change.

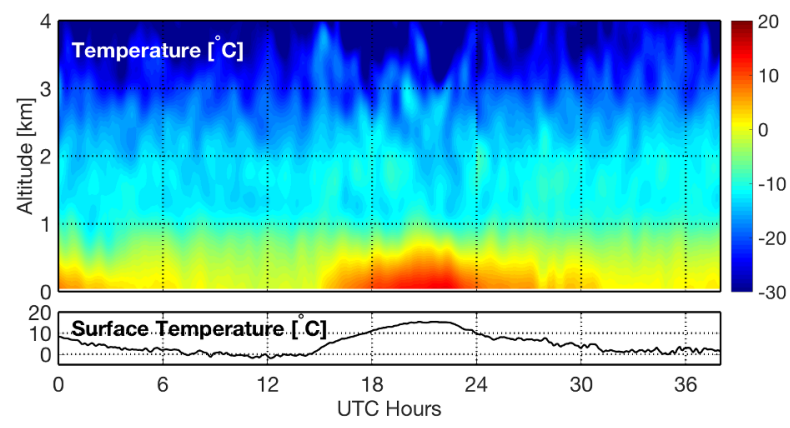

Figure 3: First temperature measurements taken with the described combined DIAL/HSRL system. Range resolution is 300 meters and temporal resolution is $\mathbf{2 0}$ minutes. First range observed is $\mathbf{3 0 0}$ meters. The first range bin here is filled with weather station data and does not come from the DIAL system.

For more qualitative comparisons, the radiosonde launched from Denver International Airport (DIA) is used to assess the vertical temperature structure. Temperature differences between the two radiosondes, launched at 12 and 36 UTC from Figure 3, and the DIAL system are shown in Figure 4. This comparison is used to assess vertical structure but is not an ideal comparison as the radiosonde launch site and DIAL are separated by $50 \mathrm{~km}$. As a result, the ground temperatures at the sites are not equal on this day. To address this issue, the vertical structure determined from the radiosonde is used with a bias applied to the 
whole profile to force ground temperatures to align.

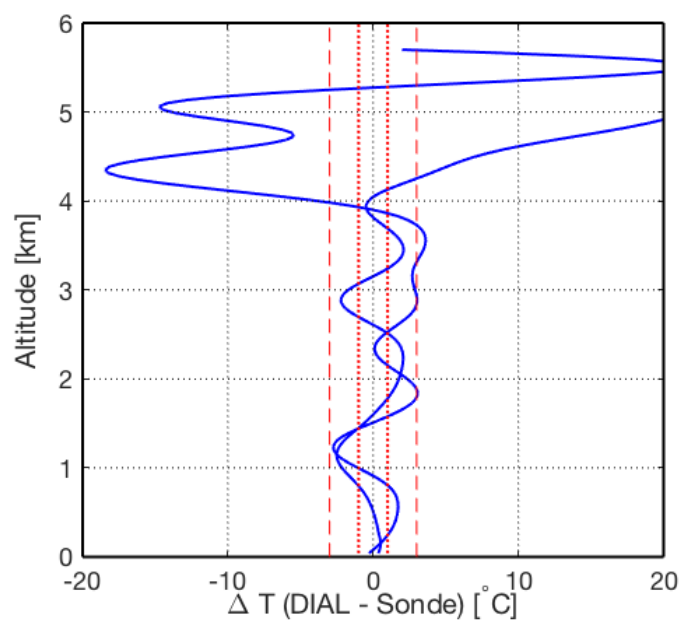

Figure 4: Comparisons of DIAL temperature data with DIA radiosonde launces. The red dashed lines are $3^{\circ} \mathrm{C}$ bounds and the dotted lines are $1^{\circ} \mathrm{C}$.

While the temperature overlap is not perfect, some promising features can be noted from this data. First, the WMO OSCAR database identifies an accuracy of $3^{\circ} \mathrm{C}$ as the beginning of useful temperature data for numerical weather prediction in the lower troposphere [14]. These data suggest that such accuracies should be possible. Second, the maximum range of the measurements shown is $4 \mathrm{~km}$. This is a direct result of low SNR measurements. Receiver optimization has increased the measured signals by a factor of approximately 5 since these data were taken.

More detailed comparisons with co-located radiosondes are planned to occur starting in April 2019. These results show promise, but need to be further investigated to understand the absolute accuracy of the designed system.

\section{CONCLUSION}

The work presented here demonstrates that DIAL systems hold promise for providing continuous monitoring of temperature in the lower troposphere. We present a design and retrieval technique that addresses previously identified issues with DIAL temperature measurements caused by molecular broadening of the backscatter spectrum. This design is a combined DIAL/HSRL system capable of measuring ancillary parameters such as water vapor mixing ratio and backscatter ratio in common volume with differential absorption measurements from oxygen. This combination along with a perturbative retrieval technique shows promise to measure atmospheric temperatures. Furthermore, given that DLB lidar systems are relatively inexpensive, and stable over periods on the order of months, this system also shows promise to be developed into a field deployable network platform. This presentation will address the development of this system. It will also demonstrate ongoing testing with this system to describe operational performance.

\section{ACKNOWLEDGEMENTS}

The National Center for Atmospheric Research is sponsored by the National Science Foundation.

\section{REFERENCES}

[1] NRC (2009): Observing Weather and Climate from the Ground Up: A Nationwide Network of Networks

[2] NRC (2010): When Weather Matters: Science and Services to Meet Critical Societal Needs

[3] NSF (2014): Dynamic Earth: GEO Imperatives \& Frontiers 2015-2020

[4] Wulfmeyer, V. et al. (2015): Rev. Geophys., 53, 819-895

[5] NASEM (2018): The Future of Atmospheric Boundary Layer Observing, Understanding, and Modeling: Proceedings of a Workshop

[6] Nehrir, A. R., et al. (2009): J. Atmos. Oceanic Technol., 20, 733-745

[7] Nehrir, A. R., et al. (2011): J. Atmos. Oceanic Technol., 28, 131-147

[8] Repasky, K. S., et al., (2013): Remote Sens., 5, 6241-6259

[9] Spuler, S. M. et al., (2015): Atmos. Meas. Tech., 8, 1073-1087

[10] Weckwerth, T. M., et al. (2016): J. Atmos. Oceanic Technol., 33, 2353-2373

[11] Hayman, M. and S. M. Spuler, (2017): Opt. Express, 25, A1096-A1110

[12] Bunn, C. E., et al. (2018): Appl. Opt., 57, 44404450

[13] Boesenberg, (1998): Appl. Opt., 37, 3845-3860

[14] WMO (2019): OSCAR: Observing Systems Capability Analysis and Review Tool. https://www.wmo-sat.info/oscar/requirements 